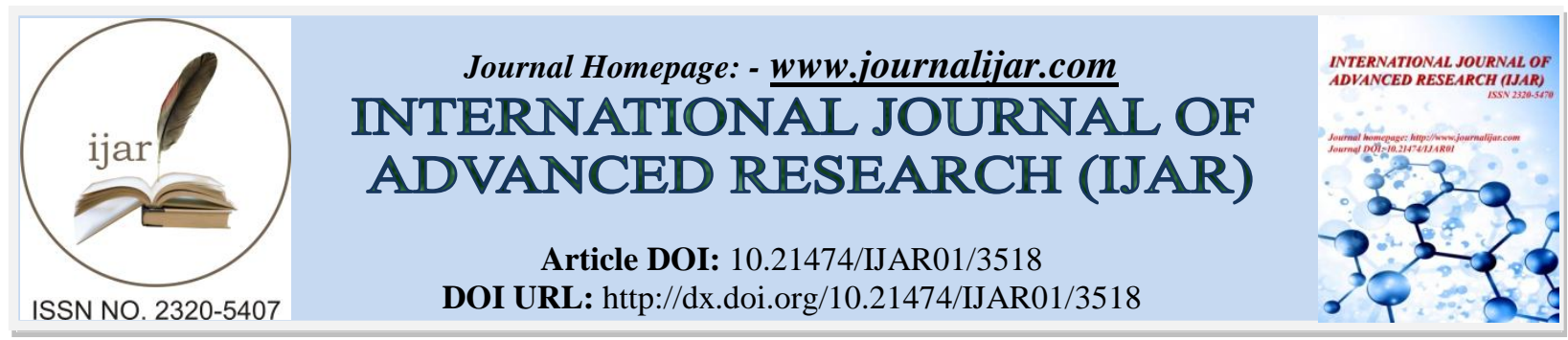

RESEARCH ARTICLE

\title{
TOBACCO CESSATION COUNSELING - THE ROLES AND RESPONSIBILITIES OF A DENTIST.
}

\section{Sudeep C B ${ }^{1}$ and Chaitra $\mathrm{T}^{2}$.}

1. Assistant Professor, Department of Public Health Dentistry, Sree Anjaneya Institute of Dental Sciences, Kerala, India.

2. Private Practitioner, Kozhikode, Kerala, India

\section{Manuscript Info}

\section{Manuscript History}

Received: 18 January 2017

Final Accepted: 20 February 2017

Published: March 2017

\section{Key words:-}

Oral Health, Tobacco, Smoking

cessation, Risk behaviors, Cancer,

Tobacco-related diseases

\begin{abstract}
India is the fourth-largest consumer of tobacco in the world and the third-largest producer of tobacco after China and Brazil. There are about 250 million tobacco users in India who account for about 19\% of the world's total 1.3 billion tobacco users. India's tobacco problem is made more complex by the fact that tobacco is used in various forms in different parts of the country. Where research once concentrated on tobacco-related diseases and their treatments, emphasis has been shifting toward examining underlying behaviors. Understanding risk profiles helps determine where and how treatment should be focused and which therapies are most effective. It is for this reason that oral health team members are in an ideal position to give their patients specific, authoritative information concerning the adverse oral effects of tobacco use and the means to tackle it. Optimizing the implementation of routine tobacco cessation intervention in general dental practice remains a key aim in the delivery of dental care.
\end{abstract}

Copy Right, IJAR, 2017,. All rights reserved.

\section{Introduction:-}

Tobacco use can lead to death, as it is one of the causes for chronic diseases. Such deaths are preventable. In 2005, tobacco use was responsible for more than 5 million deaths around the world and this number is expected to rise to 10 million deaths annually by 2020. [1] India is the fourth-largest consumer of tobacco in the world and the thirdlargest producer of tobacco after China and Brazil. There are about 250 million tobacco users in India who account for about $19 \%$ of the world's total 1.3 billion tobacco users. [2]

India's tobacco problem is made more complex by the fact that tobacco is used in various forms in different parts of the country. The prevalence of all types of tobacco use among men is about 47\% (11\%-79\% in different states) and, among women, smokeless tobacco use varies between $0.2 \%$ in Punjab and 61\% in Mizoram (Where the prevalence of women's smoking reaches a high of 22\%). Among school-going children in grades 8 through 10, current tobacco use varies from $2.7 \%$ in Himachal Pradesh to $63 \%$ in Nagaland. [3]

In India, at least 800,000 deaths every year are related to tobacco use $(700,000$ of them due to smoking). Conservative costs of treating selected tobacco-related cancers, heart and lung disease amounted to Rs 30,833 crores (US\$ 7.2 billion) in 2002-2003. [2]

Corresponding Author:- Sudeep C B.

Address:- Assistant Professor, Department of Public Health Dentistry, Sree Anjaneya Institute of 
Over the past few years, India has demonstrated a resolve to deal with the issue of tobacco use. In 2003, the Indian Parliament passed the Tobacco Control Act and in 2004 it ratified an international treaty-the World Health Organization's Framework Convention on Tobacco Control (WHO-FCTC). Efforts are urgently needed at the implementation level to reduce the current and projected harm caused by tobacco. [4]

\section{Tobacco And Oral Health:-}

Since the early 1970s, dental professionals have become increasingly aware of the damage that smoked and smokeless tobacco causes to tissues in and around the oral cavity.[5] Doubts about tobacco's adverse effects on oral health are evaporating in the face of evidence from a large and growing body of scientific literature that establishes it as an underlying cause of numerous diseases.

Ranging from mild to life-threatening, the following tobacco-related oral conditions may develop: halitosis, hairy tongue, dental calculus, periodontal disease, acute necrotizing ulcerative gingivitis, abrasion, discoloration of teeth and restorative materials, miscellaneous tissue changes, delayed wound healing, sinusitis, leukoplakia, and oral cancer. [6] Additionally, tobacco use increases and complicates treatment risks by compromising the prognosis for periodontal and other oral diseases and increasing the likelihood of the occurrence and reoccurrence of mouth cancers.[7]

Indeed, in light of current knowledge, mechanical and pharmaceutical treatment of oral diseases without addressing patient smoking is akin to medicating and binding a sliver in the hand but not removing it. The source of the problem must be managed. For e.g., treating tobacco use and dependence is as important to success in caring for periodontal disease as are restorative measures for dental caries, and preventing initiation of tobacco use by youths is as fundamental to the prevention of periodontal disease as providing fluoride and sealants are to caries prevention.[8] Where research once concentrated on tobacco-related diseases and their treatments, emphasis has been shifting toward examining underlying behaviors. Understanding risk profiles helps determine where and how treatment should be focused and which therapies are most effective. [9]

It is for this reason that oral health team members are in an ideal position to give their patients specific, authoritative information concerning the adverse oral effects of tobacco use and the means to tackle it. [10] Moreover, dentists who implement an effective smoking cessation program in their practices can expect to achieve quit rates up to 1015 percent each year among their patients who smoke or use smokeless tobacco.[11]

These rates will rise further if additional help is harnessed for smoking cessation counseling by referral to smoker's clinics and by appropriate use of pharmacotherapy. Thus, optimizing the implementation of routine smoking cessation intervention in general dental practice remains a key aim in the delivery of dental care. [12]In this regard, the 1996 clinical practice guideline Smoking Cessation recommended methods based on more than 3,000 clinical studies in several countries that had been published in peer-reviewed journals over a fifteen- year period. [13]

The guideline became the most successful of a long series produced by the Agency for Health Care Policy and Research (now the Agency for Healthcare Research and Quality), and in four years, more than 1 million copies were distributed worldwide. The 1996 guideline focused primarily on individual patient encounters. With rapid advances in the science of tobacco treatment, the 3,000 studies initially available had expanded to more than 6,000 by 1999.[14]

The 2000 guideline, Treating Tobacco Use and Dependence, placed an emphasis on regarding tobacco use as a chronic disease. Repeated care is needed to manage the long-term effects and the slow, partial recovery process. Relapse is almost inevitable during recovery, so clinician monitoring and reinforcement are needed just as is done when treating periodontal diseases, diabetes, hypertension, and other conditions requiring sustained management.[15]

The new guideline addresses issues commonly confronted, such as triage, care of special patients, and the special conditions, policies, and procedures needed to nurture effective patient cessation and maintenance services. Key recommendations in the current guideline are as follows: 
1. Tobacco dependence is a chronic condition that often requires repeated intervention. However, effective treatments exist that can produce long-term or even permanent abstinence.

2. Because effective tobacco dependence treatments are available, every patient who uses tobacco should be offered at least one of these treatments:

a. Patients willing to try to quit tobacco use should be provided with treatments identified as effective in the guideline.

b. Patients unwilling to try to quit tobacco use should be provided with brief interventions designed to increase their motivation to quit.

3. It is essential that clinicians and health care delivery systems (including administrators, insurers, and purchasers) institutionalize the consistent identification, documentation, and treatment of every tobacco user seen in a health care setting.

4. Brief tobacco dependence treatment is effective, and every patient who uses tobacco should be offered at least brief treatment.

5. There is a strong dose-response relationship between the intensity of tobacco dependence counseling and its effectiveness. Treatments involving person-to-person contact (via individual, group, or proactive telephone counseling) are consistently effective, and their effectiveness increases with treatment intensity (i.e., minutes of contact).

6. The following three types of counseling and behavioral therapies were found to be especially effective and should be used with all patients attempting tobacco cessation:

a. Provision of practical counseling (problem solving/skills training);

b. Provision of social support as part of treatment (intra-treatment support);

c. Help in securing social support outside of treatment (extra-treatment social support).

7. Numerous effective pharmacotherapies' for smoking cessation now exist. Except in the presence of contraindications, these should be used with all patients attempting to quit smoking.

a. Five first-line pharmacotherapies' were identified that reliably increase long-term smoking abstinence rates: bupropion SR, nicotine gum, nicotine inhaler, nicotine nasal spray, and nicotine patch.

b. Two second-line pharmacotherapy's were identified as efficacious and may be considered by clinicians if first-line pharmacotherapy's are not effective: clonidine and nortriptyline.

c. Over-the-counter nicotine patches are effective relative to placebo, and their use should be encouraged.

8. Tobacco dependence treatments are both clinically effective and cost-effective relative to other medical and disease prevention interventions. As such, insurers and purchasers should make sure that:

a. All insurance plans include as a reimbursed benefit the counseling and pharmacotherapeutic treatments identified as effective in the guideline; and

b. Clinicians are reimbursed for providing tobacco dependence treatment just as they are for treating other chronic conditions.

\section{Role Of The Dentist ${ }^{[16]}$}

In the clinic, dentists have an important role in helping patients quit tobacco and, at the community and national levels, to promote tobacco prevention and control strategies.

\section{Dentists in the clinics:-}

Can check for the harmful effects of tobacco in the oral cavity, they are in an ideal position to counsel patients, can target young children and youth as patients and can influence them to adopt a tobacco-free lifestyle, Can treat women of childbearing age and can inform them of the dangers of tobacco use during pregnancy. They spend more time with patients than other clinicians and use this time to counsel tobacco users to quit and reinforce messages given to patients by physicians and other caregivers about the dangers of tobacco use and the need to quit.

\section{Dentists in community and nation:-}

They can act as role models by not using tobacco or by quitting successfully. Tobacco use by dentists is a significant barrier to tobacco cessation counseling. They are also in a position to speak with authority in the community about the dangers of tobacco use; for example, the need to curb tobacco use in public and educate children about the dangers of tobacco use. Thus, they can be effective advocates for tobacco control in the community.

\section{Behavioral Counseling:-}

Counseling is a collaborative process in which the counselor assists the client in facilitating behavior change, enhancing coping skills, promoting decision making and improving relationships. [17] Tobacco use decreases when 
patients receive counseling from a health care provider. Physicians and other primary health care personnel are valuable and effective in the management of individuals with tobacco cessation. The physician has the influence, as a credible expert in a position of authority, to suggest and advise patients to quit tobacco use. [18]

Patients, who are willing to quit tobacco, should be provided with treatments identified as effective. However, for those who are unwilling to try and quit tobacco, interventions should be designed to increase their motivation to quit. [19]Under these circumstances, patients undergo a series of stages of willingness and preparedness to quit. At each stage a person is thinking and feeling differently about the tobacco use habit. These 5 stages are described below which can be categorized as: [20]

Stage-1) Pre-contemplation

Here a person is not thinking about quitting, they are not interested in change. This is because:

i) They do not see their smoking habit as a problem.

ii) They think that Tobacco is infact helpful and beneficial, that it helps them in concentration, in digestion, or for relaxing.

iii) They may be fully aware of the risks but may value tobacco use for other reasons that thus do not wish to stop.

iv) Unaware of help available and previous failed attempts at changing make them believe they cannot stop.

Stage-2) Contemplation

Here the patient knows the risks and problems, but is undecided and is unaware of the benefits on quitting smoking. In this stage the person is torn two ways, i.e., aware that he ought to stop but still feeling attached or drawn to smoking.

Stage-3) Preparation stage

Here the patient expresses a desire to quit. Those in preparation stage are planning to take action soon. They are beginning to make small changes and trying out different ways of behaving. They may tell others about their intention to stop and make clear plans on how they are going to do it.

Stage- 4) Action stage

Here patient makes visible changes and puts considerable effort for leaving the habit. This is often the time when they seek professional help.

Stage-5) Active period

During this stage, quit status is continued and strengthened, temporary stoppage becomes part of a more settled pattern. Unless this takes place the person may move into relapse, and return from there to pre-contemplation or contemplation stage.

It may be noted that a person may move from one stage to another in the forward or backward direction and may require continuous effort to move towards the direction to quit smoking. The emphasis of all the above points is to make the patients aware that tobacco use is affecting their physical health, family and social life. The guiding principle here should be to involve the patient in discussion and providing information on the consequences and risks of tobacco use.[21]

\section{Barriers to tobacco cessation:-}

A number of barriers to the provision of tobacco cessation services at a dental clinic have previously been reported. These include practitioner opinions, attitudes and perceptions toward tobacco cessation counseling.[13] Other reported barriers include lack of time, and concern about upsetting the patient150 and lack of reimbursement. [22] Although, the most significant barrier still remains a lack of education of dentists and hygienists on cessation activities during their formative years of training. [23]

Nevertheless, there are a number of important activities that could hamper this: [24]

1. Training of more dental students, dental hygienists, and dental practitioners to provide tobacco cessation counseling;

2. Increase in the number of dental practices routinely monitoring tobacco use and providing tobacco cessation programs; 
3. Increase in the utilization of the available procedures for tobacco cessation, (whether it is a covered service or not) so as to familiarize insurance companies with this practice; and

4. Stimulating the demand for more tobacco cessation coverage by employees. Such activities could go a long way towards encouraging employers to cover tobacco cessation without any differentiation by provider, as long as the outcomes are reasonably successful.

\section{Conclusion:-}

Tobacco imposes a colossal burden of disease and death leading to catastrophic health, social, economic and environmental effects. Prevalence and practices of tobacco use in India are varied and disparate. Tobacco consumption continues to grow at $2-3 \%$ per annum, and by 2020 it is predicted that it will account for $13 \%$ of all deaths in the country. [25] Tobacco dependence displays many features of a chronic disease. Only a minority of tobacco users achieve permanent abstinence in an initial quit attempt. The majorities of users persist in tobacco use for many years and typically cycle through multiple periods of remission and relapse. A failure to appreciate the chronic nature of tobacco dependence impedes clinicians' consistent assessment and treatment of the tobacco user over time. [26]

Effective interventions exist that can produce long-term cessation at up to double the rate achieved by smokers without treatment. It is important for clinicians to know that assessing and treating tobacco use generally leads to greater patient satisfaction with health care. Members of the dental profession have a unique opportunity to influence tobacco use by their patients.[27]

Dentists are obligated to help patients quit because tobacco use is the most common underlying cause of periodontal and many other oral diseases and conditions, apart from the risks to quality of life and to life itself. [28]Evidence is overwhelming that tobacco damages oral health and that clinical cessation methods work in dental practice. Although not specifically addressed in any guideline, professional education institutions, including dental and dental hygiene schools, need to identify tobacco use among all patients.[29]

The time that the dental team spends with patients usually exceeds that occurring in medical practice. The very brief (i.e., less than three minute) intervention clinicians should use to advise and assist patients need not be dedicated time but can be easily woven into other diagnostic and treatment services. As with other chronic diseases, repeated assistance is useful. Unlike treatment of other conditions, such support is particularly important to counter incentives by a powerful industry that constantly attempts to encourage previous users to relapse. [30]

Patients who quit must learn how to cope with environmental triggers. Resisting the rituals surrounding tobacco use, resisting offers of tobacco and encouraging support from those who live, work, and play with the patient are at least as important as using pharmacotherapy's for reducing discomfort associated with withdrawal and recovery. Human interaction with a respected health care provider has a powerful influence and can be tailored to individual patient interests, conditions, and culture. Pharmacotherapy should be reinstated as often as needed, that is, as often as relapsing patients are willing to make another attempt to quit, because tobacco dependence is a chronic, relapsing condition that usually requires multiple attempts before long-term abstinence is achieved. [31]

Repeated contacts and a longer duration of contact are often more easily provided in dental practice than in many medical care environments. Compensation for cessation services in dental practice may be by patient payment, by integration into another dental service, or as part of a reimbursement program. [32] Effective interventions exist that can produce long-term cessation at up to double the rate achieved by smokers without treatment. It is important for clinicians to know that assessing and treating tobacco use generally leads to greater patient satisfaction with health care. Members of the dental profession thus have a unique opportunity to influence tobacco use by their patients. 


\section{References:-}

1. Puscinkar E, Gonecka D. Tobacco smoking and the fight against tobacco addiction: A look at Iran and the other countries of the Persion Gulf Region. Pneumonol Alergal Pol. 2011; 79: 1-2.

2. Chaly PE. Tobacco control in India. Indian J Dent Res 2007; 18: 2-5.

3. Rao V, Chaturvedi P. Tobacco and health in India. Indian J Cancer 2010; 47: 3-8.

4. Collective expert report. Tobacco understanding dependence in order to act. Summary and Recommendations. INSERM.

5. Christen AG. The clinical effects of tobacco on oral tissue. J Am Dent Assoc 1990; 81: 1378-82.

6. Jones RB. Tobacco or Oral health. J Am Dent Assoc 2000; 131: 1130-36.

7. Christen AG. Tobacco cessation, the dental profession and the role of dental education. J dent edu 2011; 65: 368-374.

8. Fiona MC et al. Treating tobacco use and dependence. US Department of health and human services.2008

9. Mecklenberg RE. Tobacco prevention and control in dental practice: The future. J Dent Edu 2001; 65: 375-84.

10. Russell M A H et al. Targeting heavy smokers in general practice: randomised controlled trial of transdermal nicotine patches. BMJ 1993; 306: 1308-12.

11. Warnakulasuriya S. Effectiveness of tobacco counselling in the dental office. J Dent Educ 2002; 66: 1079-87.

12. Raymond E et al. What's new in smoking cessation: Zyban. Can Fam Physician. 1999; 45: 633-4.

13. Yip JK et al. Dental students' attitudes towards smoking cessation guidelines. J Dent Educ 2000; 64: 641-50.

14. Tobacco or health: A global status report. WHO publications: Geneva; 1997.

15. Crane J, Blakely T, Hill S. Time for major roadworks on the tobacco road? NZ Med J 2004; 117: 801-04.

16. Sudeep CB. Tobacco Cessation Counseling - The Inside Story.Coorg Dental Times. 2012 July (Issue 2) : 4-9.

17. Lennox A S, Taylor R J. Factors associated with outcome in unaided smoking cessation, and a comparison of those who have never tried to stop with those who have. Br J Gen Pract. 1994; 44: 245-250.

18. Balanda K P, Lowe J B, Fleming M. Comparison of two self-help smoking cessation booklets. Tob Control. 1999;8: 57-61.

19. Helstrom A, Hutchison K, Bryan A. Motivational enhancement therapy for high-risk adolescent smokers. Addict Behav. 2007 ;32:2404-10.

20. Hughes J. An algorithm for choosing among smoking cessation treatments. J Sub Abuse Treatment 2008; 34 : 426-432.

21. Levinson $\mathrm{A} \mathrm{H}$ et al. Tailored behavioral support for smoking reduction: development and pilot results of an innovative intervention. Health Educ Res. 2008; 23:335-46.

22. Campbell HS, Stattan M, Pettery T. Patient perceptions of tobacco cessation services in dental offices. J Am Dent Assoc 1999; 130: 219-26.

23. Weaver RG et al. Tobacco control and prevention effort in dental education. J Dent Educ 2002; 66: 426-29.

24. Damiano PC. The question of cost: Reimbursement and Remuneration. J Dent Educ 2001; 65: 364-67.

25. Jettle AM, Feldman HA, Tennsladt SL. Tobacco use: A modifiable risk factor for dental disease among the elderly. Am J Pub Health 1993;9:1271-76.

26. Jha, P., F. J. Chaloupka. "Tobacco Addiction." In Disease Control Priorities in Developing Countries, 2nd ed. Oxford university Publication.2000.

27. Chua H F et al. Self-related neural response to tailored smoking-cessation messages predicts quitting. Nat Neurosci. $2011 ; 14:$ 426-427.

28. Benowitz NL. Nicotine Addiction. N Engl J Med 2010; 362: 2295-2303.

29. Jandoo T, Mehrotra R. Tobacco control in India: present scenario and challenges ahead. Asian Pacific J Cancer Prev 2008; 9: 805-10.

30. O'Loughlin J, Gervais A, Dugas E, Meshefedjian G. Milestones in the process of cessation among novice adolescent smokers. Am J Public Health. 2009;99:499-504.

31. Gonzales D et al. Immediate vs Delayed quitting and rates of relapse among smokers treated successfully. Addiction 2010; 105: 2002-13.

32. Volpp K G et al. A randomized, controlled trial of financial incentives for smoking cessation. N Engl J Med. 2009 ;360(7):699-709. 